\title{
Comments on the status of modern covariance data based on different fission and fusion reactor studies
}

\author{
Ivan Kodeli* \\ Jožef Stefan Institute, Jamova 39, Ljubljana, Slovenia
}

Received: 29 September 2017 / Received in final form: 6 February 2018 / Accepted: 14 May 2018

\begin{abstract}
Both the availability and the quality of covariance data improved over the last years and many recent cross-section evaluations, such as JENDL-4.0, ENDF/B-VII.1, JEFF-3.3, etc. include new covariance data compilations. However, several gaps and inconsistencies still persist. Although most modern nuclear data evaluations are based on similar (or even same) sets of experimental data, and the agreement in the results obtained using different cross-sections is reasonably good, larger discrepancies were observed among the corresponding covariance data. This suggests that the differences in the covariance matrix evaluations reflect more the differences in the (mathematical) approaches used and possibly in the interpretations of the experimental data, rather than the different nuclear experimental data used. Furthermore, "tuning" and adjustments are often used in the process of nuclear data evaluations. In principle, if adjustments or "tunings" are used in the evaluation of cross-section then the covariance matrices should reflect the cross-correlations introduced in this process. However, the presently available cross-section covariance matrices include practically no cross-material correlation terms, although some evidence indicate that tuning is present. Experience in using covariance matrices of different origin (such as JEFF, JENDL, ENDF, TENDL, SCALE, etc.) in sensitivity and uncertainty analysis of vast list of cases ranging from fission to fusion and from criticality, kinetics and shielding to adjustment applications are presented. The status of the available covariance and future needs in the areas including secondary angular and energy distributions is addressed.
\end{abstract}

\section{Introduction}

The performance of the new cross-section evaluations, if judged by the agreement with the large set of integral experiments, greatly improved over the last decades. Indeed, using the recent nuclear data evaluations, the calculation-to-experiment $(\mathrm{C} / \mathrm{E})$ ratios for the large series of critical integral benchmarks are indeed excellent. For example, the comparison presented in [1] reveals that almost $50 \%$ of the calculated $k_{\text {eff }}$ values (about 900 out of over 2000 critical benchmarks analysed using ENDF/BVII.1, JENDL-4.0 and JEFF-3.1.1) lie within one standard deviation $(1 \sigma)$ of the experimental plus MCNP statistical uncertainty. However, such good agreement of $\mathrm{C} / \mathrm{E}$ is difficult to understand from the mathematical (statistical) point of view. Indeed, much larger dispersion of results is to be expected from the statistical point of view taking into account in addition also the calculational uncertainties due to nuclear data, unless (1) the later are very small (highly unlikely), or (2) are correlated with the integral

\footnotetext{
* e-mail: ivan.kodeli@ijs.si
}

results, suggesting some adjustment or tuning procedure was used in the evaluation process. Manifestly, these "tunings" are not reflected in the cross-section covariance matrices, which include practically no cross-material correlation terms, with the exception of cross-correlations between $(n, f)$ reactions of $\mathrm{U}$ and $\mathrm{Pu}$ isotopes in the JENDL evaluations ( -3.3 and on) [2]. The total uncertainty to cover $68 \%$ of the 2000 analysed $\mathrm{C} / \mathrm{E}$ cases is around $1.8 \sigma$ of the experimental uncertainty, which would correspond to the average $1 \sigma$ computational uncertainty of only around $500 \mathrm{pcm}$, i.e. of a similar order of magnitude as the measurement uncertainties and lower than the typically calculated values.

\section{SUSD3D and XSUN-2017 computer code package}

The SUSD3D [3] code was developed in the 1990s to allow $1-, 2-$, and 3-dimensional cross-section sensitivity and uncertainty calculations. In the past few decades the code was applied to waste range of different nuclear applications, including neutron and gamma ray shielding, 
criticality, and kinetics. The latest version of SUSD3D is part of the XSUN-2017 [4] code package.

An important factor limiting the use of $\mathrm{S} / \mathrm{U}$ analysis is the availability and the quality of cross-section covariance data. Several tools and nuclear data libraries were developed to facilitate the access and allow the validation of the data. This will be presented in Section 3 .

\subsection{Examples of applications}

The SUSD3D code was used since early 1990s for very various applications, such as:

- reactor pressure vessel surveillance dosimetry [3]: uncertainty in predicted dosimeter reaction rates and pressure vessel exposition, determination of realistic safety margins and consequently the reactor lifetime predictions;

- fission shielding benchmarks [3]: sensitivity and uncertainty in the measured reaction rates were calculated for the several benchmarks from the SINBAD database, such as the ASPIS Iron, ASPIS Iron88 and VENUS-3 pressure vessel dosimetry benchmark;

- sensitivity/uncertainty pre- and post-analysis of the fusion shielding benchmarks performed at the Frascatti Neutron Generator (FNG) at ENEA Frascatti (sensitivity/uncertainty of the measured fast/thermal activation rates and the tritium production in FNG-Bulk Shield benchmark, FNG-Streaming, FNG-SiC, FNG-Tungsten [5], FNG HCPB and FNG-HCLL tritium breeding modules [6,7] and FNG Copper [8,9] benchmarks);

- criticality benchmarks (sensitivity to $k_{\text {eff }}$ and $\beta_{\text {eff }}$ ): many benchmarks from IRPhE and ICSBEP (KRITZ-2 [10], SNEAK-7A and -7B [11], VENUS-2, etc.), MYRRHA reactor [12], etc.;

- oil well logging: sensitivity and uncertainty of the carbonto-oxygen gamma-ray ratio [13].

\section{Types of covariance matrices}

Different data formats for cross-section covariances are available in the evaluated files according to the type of nuclear data:

$-\mathrm{MF}=31$ : covariance of average number of neutrons per fission $(\bar{v}-\mathrm{MT}=452,455,456)$;

$-\mathrm{MF}=32$ : shape and area of individual resonances;

$-\mathrm{MF}=33$ : covariance of neutron cross-section;

$-\mathrm{MF}=34$ : covariance of angular distribution of secondary neutron (SAD). NJOY processing is available for the reaction $\mathrm{MT}=251 / P_{1}$ only;

- $\mathrm{MF}=35$ : covariance of energy distribution of secondary neutron (SED). NJOY processing is available for the reaction $\mathrm{MT}=18$ only;

$-\mathrm{MF}=30$ : covariances obtained from parameter covariances and sensitivities (no NJOY processing available yet);

$-\mathrm{MF}=40$ : covariances for production of radioactive nuclei.

Several nuclear data processing codes and multigroup covariance data libraries are available from the OECD/ NEA Data Bank, such as:
- NJOY-99/-2012/-2016 (ERRORR, COVR) [14]: processing of files $\mathrm{MF}=31-35$ (COVFILS format);

- PUFF-IV: code system to generate multigroup covariance matrices from ENDF/B-VI uncertainty files (COVERX Format);

- SUNJOY/ERRORR34 (part of SUSD3D package) [3]: secondary angular distributions (SAD) covariance ( $\mathrm{MF}=4$ and 34$)$ processing code (COVFILS format);

- ANGELO-LAMBDA [15]: utility programs for interpolation and mathematical verification of the matrices (COVERX and BOXER format input data, COVFILS output format);

- Multigroup covariance data libraries: ZZ-VITAMIN-J/ COVA, ZZ-SCALE5.1/COVA and ZZ-SCALE6/ COVA-44G (44-group cross-section covariance matrix library extracted from SCALE6.0 [16]).

\subsection{Uncertainties in prompt and delayed Nu-bar $\left(\bar{v}_{p} / \bar{v}_{d}\right)(\mathrm{MF} 31)$}

The uncertainties in prompt nu-bar directly influence the uncertainty in $k_{\text {eff }}$ and are therefore often among its major contributors. Large differences can be observed among different evaluations, the standard deviations ranging from as low as $\sim 0.1 \%$ (most probably unrealistically optimistic) up to $\sim 1 \%$. Inconsistencies between the prompt and total neutron multiplicities were also found in ENDF/B-VII.1 [17]. This results in very different uncertainty estimations (see an example in Tab. 1).

Examples of $\bar{v}_{p}$ covariances of ${ }^{239} \mathrm{Pu}$ are shown in Figure 1. The standard deviations passed from $\sim 1 \%$ in older SCALE- 5.1 and $-6.0 \mathrm{~m}$ libraries to $\sim 0.1 \%$ in the recent ENDF/B-VII.1 and JENDL-4.0 evaluations, most probably to accommodate a better $\mathrm{C} / \mathrm{E}$ agreement for a large series of integral benchmarks, rather than reflecting the uncertainties in differential measurements. Whereas this approach may provide relatively realistic uncertainties in $k_{\text {eff }}$ for classes of problems covered by the integral benchmarks, it is likely to lead to biased results of adjustment analysis since preventing any modifications of $\bar{v}_{p}$ and thus freezing the values including the tunings introduced during the evaluations. Furthermore, no crossisotope correlations are included in the available evaluations. Due to their importance for burn-up calculations the fission yield correlation matrices were evaluated in [18].

Similarly, the uncertainties in delayed nu-bar were found important for reactor kinetics calculations, such as the uncertainties in effective delayed neutron fraction $-\beta_{\text {eff. }}$ Only JENDL-4.0 [2] evaluation includes the corresponding covariance matrices (Fig. 1), therefore most $\beta_{\text {eff }} \mathrm{S} / \mathrm{U}$ analyses were based on these data [11]. However, here again no correlations are proposed between delayed nu-bar values of different isotopes even if it is evident that such correlations exist because of the use of similar measurement techniques and theoretical computational model. Missing correlation in evaluated files are likely to result in misleading uncertainty calculations. Uncertainties calculated assuming no- and full-correlations between the $\bar{v}$ of different isotopes strongly vary on the cases studied (see few examples in Tab. 1). 
Table 1. Uncertainties in $k_{\text {eff }}$ and $\beta_{\text {eff }}$ calculated using the SUSD3D code. The two values for the $\beta_{\text {eff }}$ uncertainty correspond to the assumption of no/full correlation among the $\bar{v}_{d}$ uncertainties of different actinides. SAD/SED uncertainties are not included.

\begin{tabular}{lllr}
\hline Benchmark & \multicolumn{2}{l}{$\begin{array}{l}\text { Covariance } \\
\text { evaluation }\end{array}$} & \multicolumn{2}{c}{ Uncertainty (\%) } \\
\cline { 2 - 3 } & & $k_{\text {eff }}$ & $\beta_{\text {eff }}$ \\
\hline SNEAK-7A & JENDL-4.0 & $0.61 / 0.64$ & $2.7 / 3.8$ \\
& ENDF/B-VII.1 & $0.77 / 0.79$ & \\
& SCALE-6.0 m & $1.09 / 1.20$ & $2.6 / 3.3$ \\
FLATTOP-Pu & JENDL-4.0 & $0.70 / 0.72$ & \\
& ENDF/B-VII.1 & $0.55 / 0.56$ & $2.5 / 2.7$ \\
JEZEBEL & SCALE-6.0 m & $1.20 / 1.28$ & $0.60 / 0.61$ \\
& JENDL-4.0 & 0.56 & $1.35 / 1.41$ \\
\hline
\end{tabular}

An attempt to estimate the correlations among the $\bar{v}_{d}$ values of different actinides is described in [19]. The GEF code [20] was used to calculate the variance-covariance of the delayed fission yield data for ${ }^{235} \mathrm{U},{ }^{238} \mathrm{U}$ and ${ }^{239} \mathrm{Pu}$ actinides as a function of input model parameters and the corresponding uncertainties. Typical values of the correlations coefficients as high as around 0.8 between ${ }^{235} \mathrm{U}$ and ${ }^{239} \mathrm{Pu}$, and around 0.3 between ${ }^{238} \mathrm{U}$ and ${ }^{239} \mathrm{Pu}$ were observed. Is was concluded that this is likely to have considerable impact on the uncertainty propagation calculations, such as those of the effective delayed neutron fraction and the burn-up evolution [21].

\subsection{MF33 covariance matrices}

The covariance information of the type MF33 is most widely evaluated and used, also because the processing is in general well established. The main concerns represent the lack of correlations between different isotopes and rather large differences among evaluations in some cases. An example of the use of different copper and iron covariance evaluations is shown in Tables 2 and 3, respectively. More details can be found in references [8] and [9].

\subsection{SAD uncertainties (MF34)}

The importance of the uncertainties in the SAD was demonstrated in several fast neutron applications such as fusion [22], fast reactors, etc. and the processing of these data and te $\mathrm{S} / \mathrm{U}$ methodology is available and tested since decades. In the EFF-2 evaluations in the 1990s, the covariance matrices for angular distribution of secondary particles became available for the elastic cross-sections for ${ }_{56} \mathrm{Fe},{ }_{52} \mathrm{Cr},{ }_{58} \mathrm{Ni}$ and ${ }^{60} \mathrm{Ni}[23,24]$. The matrices were prepared in the file $\mathrm{MF}=34 \mathrm{ENDF} / \mathrm{B}-6$ format in terms of covariances among Legendre coefficients, and energy-dependent correlation was included as well. The evaluations included the terms from $P_{1}$ to $P_{6}$.

In the scope of the European Fusion File project in 1995 a procedure was developed to process the SAD covariance matrices into a multigroup form to be used subsequently by the SUSD3D S/U code [25]. The processing code, called ERRORR34, now part of the SUSD3D [3] code package, can process the ENDF/B-6 format files $\mathrm{MF}=4$ and 5 (SAD/SED cross-sections), and $\mathrm{MF}=34$ (SAD covariances). Group-collapse strategy similar to the one used in NJOY [14] was adopted, therefore many NJOY-91.91 (ERRORR) subroutines were used. As in the ERRORR module, union groups are first formed as an union of the user's and ENDF/B grids. The SAD partial cross-sections, weighting flux and covariance matrices are defined to produce multigroup values on this grid. The covariance matrices in the user defined energy structure are then calculated from:

$$
\operatorname{cov}\left(\sigma_{G}^{l}, \sigma_{G^{\prime}}^{l^{\prime}}\right)=\sum_{g \in G, g^{\prime} \in G^{\prime}} r \operatorname{cov}\left(\sigma_{g}^{l}, \sigma_{g^{\prime}}^{l^{\prime}}\right) \Phi_{g} \sigma_{g}^{l} \Phi_{G} \Phi_{g^{\prime}} l_{g^{\prime}}^{l^{\prime}} \Phi_{G^{\prime}},
$$

where $g$ refers to the union group, and $G$ to the user defined energy group, $\sigma_{G}^{l}$ represent the $l$ th Legendre polynomial coefficients of the SAD partial cross-sections, in energy group $G, r \operatorname{cov}\left(\sigma_{g}^{l}, \sigma_{g^{\prime}}^{l^{\prime}}\right)$ is the SAD relative covariance in union group structure, $\Phi_{G}$ is the weighting flux in group $G$. Finally the relative covariance in the new energy grid is obtained from:

$$
r \operatorname{cov}\left(\sigma_{G}^{l}, \sigma_{G^{\prime}}^{l^{\prime}}\right)=\operatorname{cov}\left(\sigma_{G}^{l}, \sigma_{G^{\prime}}^{l^{\prime}}\right) \sigma_{G}^{l} \cdot \sigma_{G^{\prime}}^{l^{\prime}}
$$

Modifications were subsequently needed also in the SUSD3D code, in order to take into account the full covariance matrix information provided by ERRORR34.

Among the recent nuclear data evaluations, the JENDL-4.0 [2] includes the SAD (MF34) covariances relative to the reaction type MT251 (average scattering cosine) for several important isotopes (Fe, $\mathrm{U}, \mathrm{Pu}$, etc.). The recent versions of NJOY (NJOY-99, -2012 and -2016) can also process these data in the multigroup form. Note however that these data (and the NJOY processing) is of course limited to the $P_{1}$ Legendre term. MT34/MF251 


\section{SCALE-6.0m}
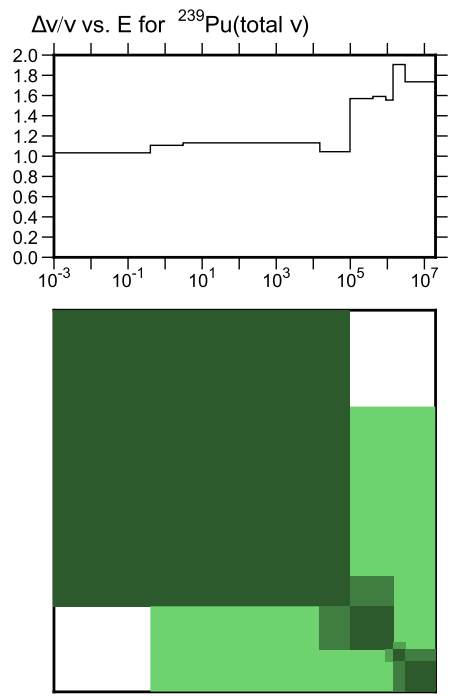

Correlation Matrix

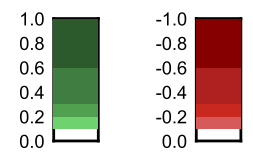

JENDL-4.0 $\nu_{\text {prompt }}^{-}$

$\Delta$ v/v vs. E for ${ }^{239} \mathrm{Pu}($ prompt v)
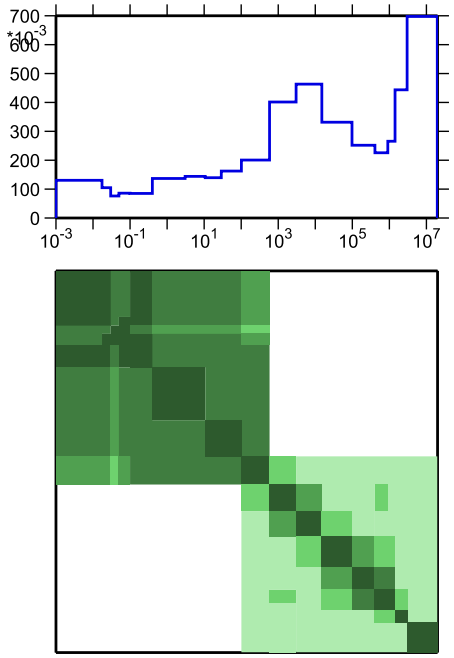

Correlation Matrix
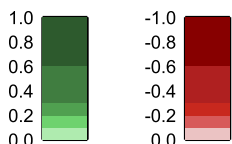

Linear Axes:

Rel. Standard Dev. (\%)

Logarithmic Axes:

Energy (eV)

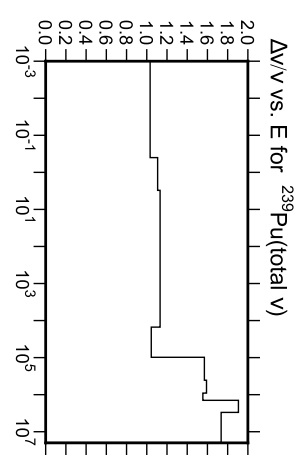

Ordinate scales are $\%$ relative standard deviation and nu-bar.

Abscissa scales are energy (eV)

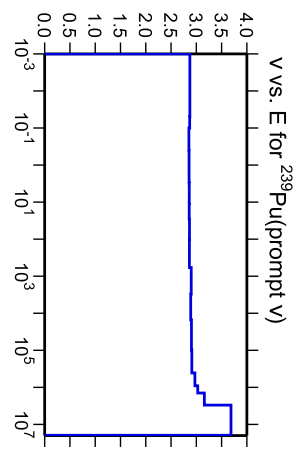

ENDF/B-VII.1

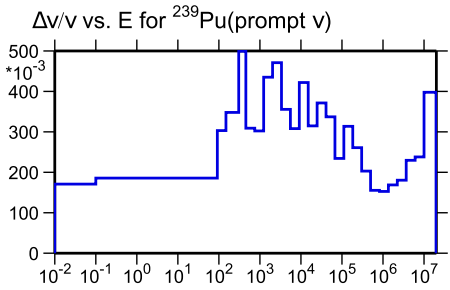

Ordinate scales are $\%$ relative standard deviation and nu-bar.

Abscissa scales are energy (eV).
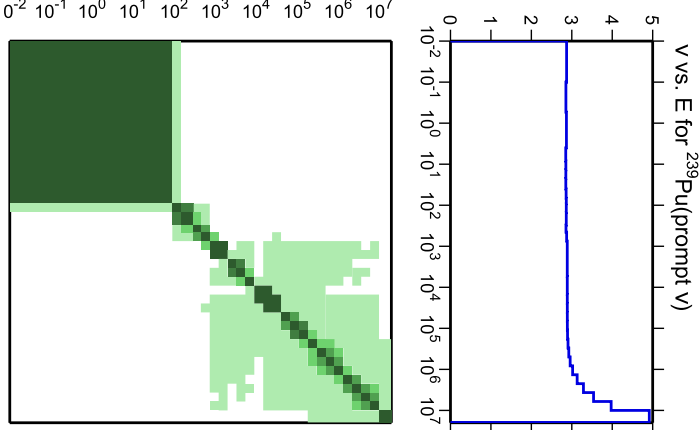

Correlation Matrix

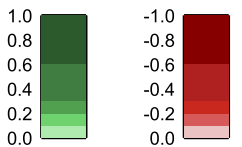

JENDL-4.0 $\nu_{\text {delayed }}$

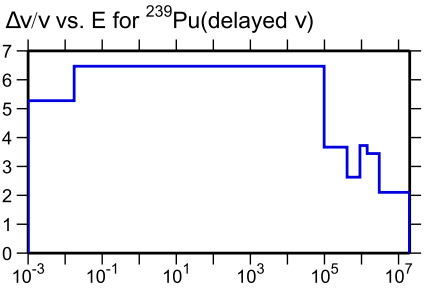

Ordinate scales are $\%$ relative standard deviation and nu-bar.

Abscissa scales are energy $(\mathrm{eV})$.
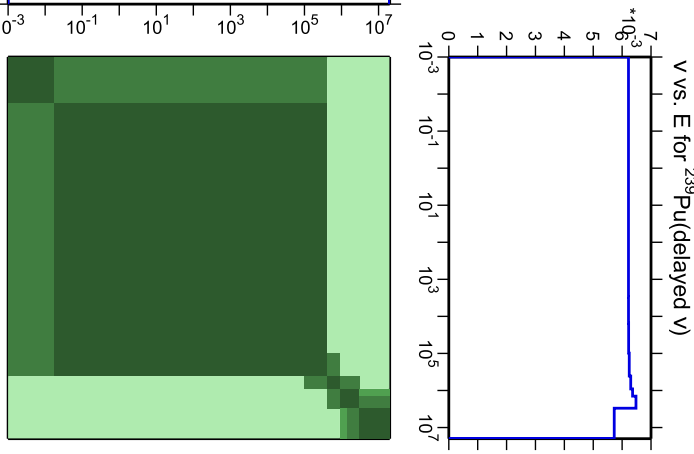

Correlation Matrix

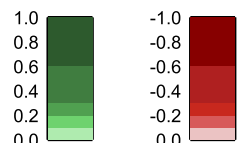

Fig. 1. Covariance matrices of ${ }^{239} \mathrm{Pu}$ for $\bar{v}$ from the SCALE- $6.0 \mathrm{~m}, \mathrm{ENDF} / \mathrm{B}-\mathrm{VII} .1$ and JENDL-4.0 evaluations.

covariances for few isotopes $\left({ }^{56} \mathrm{Fe}\right)$ are likewise included in the ENDF/B-VII.1 [26] evaluation. Even more SAD covariances are available in the TENDL [27] libraries for elastic and some inelastic reactions. The evaluations include also higher than $P_{1}$ Legendre terms, however only
$P_{1}$ can be processed using the recent NJOY ( -99 and above) codes. The ERRORR34 code sequence can not be used in these cases since it is based on the older NJOY-91 version and needs to be updated (e.g. to the NJOY-2016 version) for this purpose. 
Table 2. FNG-Cu benchmark: uncertainty due to transport cross-sections of different origin compared to the $\mathrm{C} / \mathrm{E}$ values.

\begin{tabular}{rllll}
\hline $\begin{array}{l}\text { Reaction rate } \\
\text { and det. position }\end{array}$ & \multicolumn{3}{c}{ Uncertainty $(\%)$} & C $/$ E \\
\cline { 2 - 4 } & JEFF-3.2 & ENDF/B-VI.8 & TENDL-2013 & \\
\hline${ }^{58} \mathrm{Ni}(n, p)-35 \mathrm{~cm}$ & 5.2 & 13.7 & 22.9 & $1.03 / 0.98$ \\
$-57 \mathrm{~cm}$ & 9.9 & 27.2 & 41.9 & $1.03 / 0.91$ \\
${ }^{115} \mathrm{In}(n, n)-35 \mathrm{~cm}$ & 5.1 & 9.4 & 12.1 & $0.78 / 0.68$ \\
$-57 \mathrm{~cm}$ & 8.9 & 18.7 & 23.5 & $0.69 / 0.54$ \\
${ }^{27} \mathrm{Al}(n, \alpha)-57 \mathrm{~cm}$ & 13.1 & 33.2 & 51.9 & $0.88 / 0.77$ \\
${ }^{93} \mathrm{Nb}(n, 2 n)-57 \mathrm{~cm}$ & 13.8 & 34.7 & 53.4 & $0.92 / 0.79$ \\
${ }^{197} \mathrm{Au}(n, \gamma)-57 \mathrm{~cm}$ & Processing & 19.9 & 18.6 & $0.58 / 0.63$ \\
${ }^{186} \mathrm{~W}(n, \gamma)-57 \mathrm{~cm}$ & error & 28.6 & 27.3 & $0.41 / 0.37$ \\
\hline
\end{tabular}

Table 3. ASPIS IRON-88 benchmark: computational $(\Delta C)$ vs. experimental $(\Delta E)$ uncertainties.

\begin{tabular}{|c|c|c|c|c|c|c|c|}
\hline \multirow[t]{2}{*}{ Reaction and position } & & \multicolumn{4}{|c|}{$\Delta C \mathrm{SAD}(\%)$} & \multirow{2}{*}{$\begin{array}{l}\Delta C \text { Total } \\
\text { ENDFB7.1/JENDL4/ } \\
\text { TENDL2015 }\end{array}$} & \multirow[t]{2}{*}{$\Delta E(\%)$} \\
\hline & & EFF-2.4 & $\mathrm{ENDF} / \mathrm{B} 7.1$ & JENDL4 & TENDL2015 & & \\
\hline \multirow[t]{3}{*}{${ }^{32} S(n, p)$} & A7 & 1.3 & 1.3 & 2.9 & & $12 / 17$ & 6.5 \\
\hline & A12 & 2.2 & 2.1 & 6.0 & 51 & $21 / 35 / 33$ & 6.5 \\
\hline & A14 & 2.5 & 2.3 & 7.2 & 60 & $25 / 43 / 40$ & 8.6 \\
\hline \multirow[t]{2}{*}{${ }^{115} \operatorname{In}\left(n, n^{\prime}\right)$} & A7 & 0.6 & 0.6 & 2.3 & & $11 / 15$ & 4.5 \\
\hline & A11 & 0.9 & 1.0 & 3.2 & 11 & $16 / 18 / 20$ & 4.7 \\
\hline \multirow[t]{2}{*}{${ }^{103} R h\left(n, n^{\prime}\right)$} & A7 & & 0.3 & 1.0 & & $8 / 9$ & 5.1 \\
\hline & A14 & & 0.3 & 1.1 & & $20 / 16 / 26$ & 5.1 \\
\hline${ }^{27} A l(n, \alpha)$ & A7 & 3.4 & 3.4 & 1.4 & 37 & $32 / 31 /(27)$ & 4.7 \\
\hline \multirow[t]{3}{*}{${ }^{197} A u(n, \gamma)$} & A7 & 0.1 & 0.1 & 0.3 & & $10 / 9$ & 4.2 \\
\hline & A11 & 0.1 & 0.1 & 0.3 & & $9 / 9$ & 4.2 \\
\hline & A14 & 0.1 & 0.1 & 0.3 & 1.1 & $8 / 8 / 4$ & 4.2 \\
\hline
\end{tabular}

An example of the EFF-2.4 covariance matrices for ${ }^{56} \mathrm{Fe}$ (processed by the code ERRORR34) is presented in Fig. 2), compared to the recent evaluation available in the JENDL4.0, ENDF/B-VII.1 and TENDL-2015 evaluations and processed using NJOY-99. Note that contrary to the recent evaluations the EFF-2.4 data include the terms $P_{1}$ to $P_{6}$.

An example of the SAD uncertainties for the ASPISIRON88 benchmark calculated using the SUSD3D code and the above ${ }^{56} \mathrm{Fe}$ covariance matrices is given in Table 3 . Considerable spread of results can be observed, however all suggesting that SAD uncertainties cannot be neglected for high-energy reactions.

\subsection{SED uncertainties (MF35)}

Uncertainties in the Secondary Energy Distributions are at present only available for the prompt fission neutron spectra (PFNS) and relatively complete data are included in recent evaluations such as JENDL-4.0, ENDF/B-VII.1 and JEFF-3.3. However, the correletions among the covariances for different incident neutron energies are missing. The conservative assumption of total correlation is in this conditions probably the most appropriate.

Several sensitivity methods were studied in the scope of the WPEC-26, concluding with recommending the constrained sensitivity method [28].

However, covariance information for other reactions is still missing. A simple method for evaluating covariances for delayed fission spectra, which are important for the calculation of $\beta_{\text {eff }}$ uncertainty, was proposed in [11]. An approximate "two-block" covariance matrices were constructed based on a simple common sense assumption of an energy-uniform standard deviation of $15 \%$ and a complete anti-correlation between the energies above and below the 
EFF-2.4

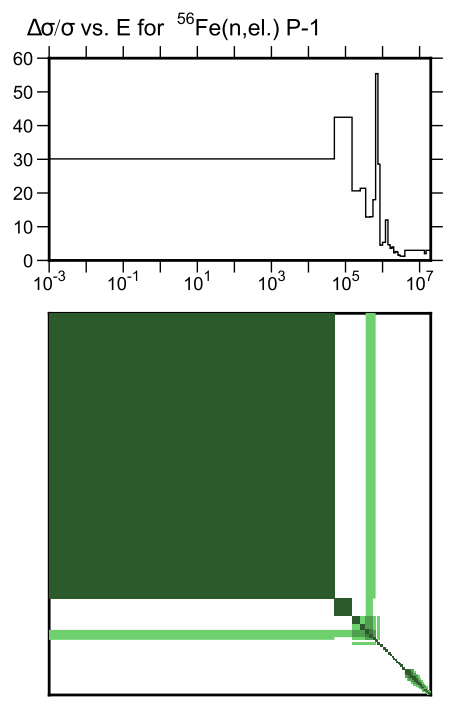

Correlation Matrix

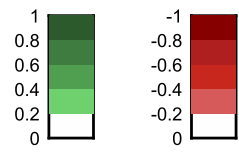

ENDF/B-VII.1
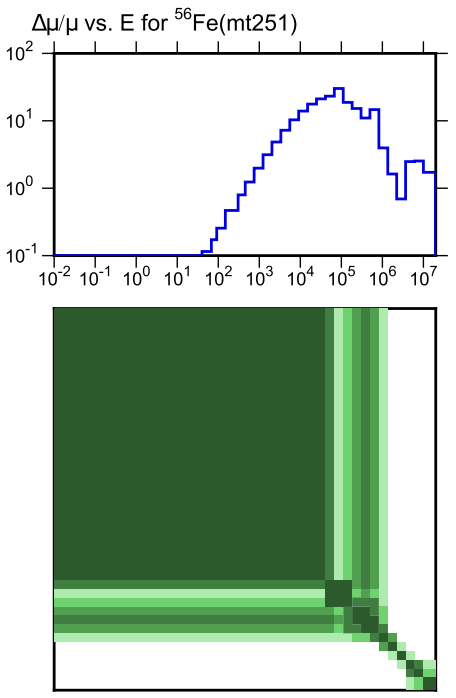

Correlation Matrix
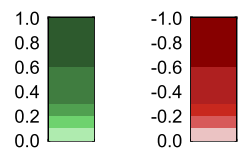

JENDL-4.0
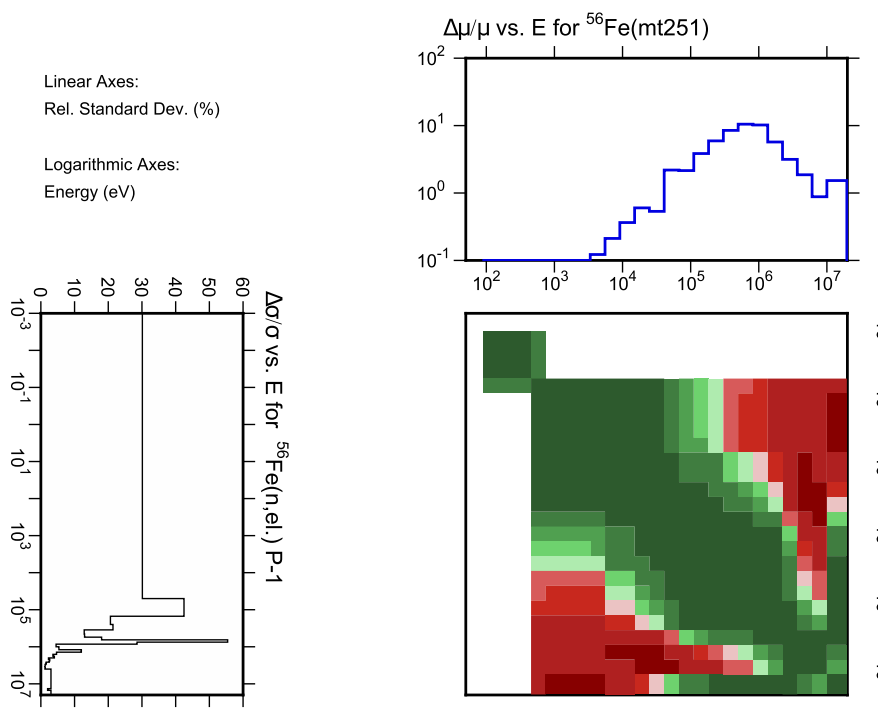

Linear Axes:

Rel. Standard Dev. (\%)

Logarithmic Axes:

Energy (eV)

Ordinate scales are $\%$ relative standard deviation and mu-bar

Abscissa scales are energy (eV).

Warning: some uncertainty data were suppressed.

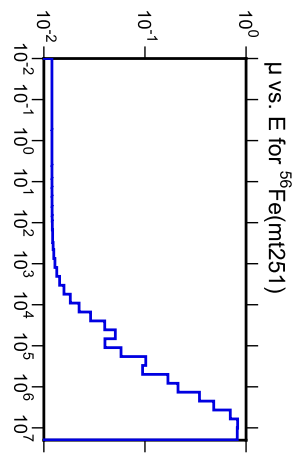

Fig. 2. SAD covariance matrices of ${ }^{56} \mathrm{Fe}$ elastic cross-sections from the EFF-2.4, JENDL-4.0, ENDF/B-VII.1 and TENDL-2015 evaluations. Warning: apparent similarity between the JENDL-4.0 and ENDF/B-VII.1 covariances is only an artifact of log/log scale which is for some reason used in the recent versions of NJOY. Switching back to the old (much more informative) lin/log presentation is strongly recommended (e.g. by redefining the "yrtest" parameter in the COVR module).

mean delayed neutron energy for each of the 6 delayed groups. Conservative assumption of the complete correlation between the 6 individual groups was adopted.
To test the validity of this method a similar procedure, except assuming a uniform $4 \%$ standard deviation instead of $15 \%$, was applied to the PFNS, where comparison with 
Table 4. Fission spectra uncertainties in $k_{\text {eff }}$ and $\beta_{\text {eff }}$ calculated using the approximate "two-block" prompt fission neutron spectra covariances (i.e. assuming flat anti-correlated $4 \%$ standard deviation) compared to those based on covariances from JENDL-4.0 and SCALE-6.0.

\begin{tabular}{|c|c|c|c|c|c|c|c|}
\hline \multirow[t]{2}{*}{ Benchmark } & \multirow[t]{2}{*}{ Isotope } & \multicolumn{3}{|c|}{$k_{\text {eff }}$ uncertainty $(\mathrm{pcm})$} & \multicolumn{3}{|c|}{$\beta_{\text {eff }}$ uncertainty $(\mathrm{pcm})$} \\
\hline & & Two-block & JENDL-4.0 & SCALE-6.0 & Two-block & JENDL-4.0 & SCALE-6.0 \\
\hline \multirow{4}{*}{ SNEAK 7A } & ${ }^{235} \mathrm{U}$ & 22 & 27 & 20 & 53 & 50 & 36 \\
\hline & ${ }^{238} \mathrm{U}$ & 71 & 99 & 78 & 49 & 25 & 17 \\
\hline & ${ }^{239} \mathrm{Pu}$ & 261 & 288 & 264 & 572 & 523 & 414 \\
\hline & Total & 271 & 305 & 276 & 577 & 526 & 416 \\
\hline \multirow{4}{*}{ SNEAK 7B } & ${ }^{235} \mathrm{U}$ & 41 & 49 & 37 & 78 & 71 & 51 \\
\hline & ${ }^{238} \mathrm{U}$ & 109 & 150 & 119 & 36 & 46 & 18 \\
\hline & ${ }^{239} \mathrm{Pu}$ & 335 & 377 & 343 & 551 & 489 & 377 \\
\hline & Total & 354 & 409 & 364 & 557 & 496 & 381 \\
\hline Jezebel & ${ }^{239} \mathrm{Pu}$ & 292 & 367 & 343 & 637 & 820 & 774 \\
\hline Skidoo Jez-23 & ${ }^{233} \mathrm{U}$ & 106 & 121 & 97 & 212 & 106 & 91 \\
\hline \multirow{4}{*}{ Popsy Flat-Pu } & ${ }^{235} \mathrm{U}$ & 6 & 8 & 6 & 28 & 30 & 22 \\
\hline & ${ }^{238} \mathrm{U}$ & 47 & 68 & 54 & 105 & 94 & 79 \\
\hline & ${ }^{239} \mathrm{Pu}$ & 302 & 371 & 348 & 100 & 172 & 45 \\
\hline & Total & 306 & 377 & 352 & 147 & 199 & 93 \\
\hline \multirow{3}{*}{ Topsy Flat-25 } & ${ }^{235} \mathrm{U}$ & 220 & 290 & 229 & 195 & 374 & 289 \\
\hline & ${ }^{238} \mathrm{U}$ & 44 & 64 & 50 & 47 & 92 & 70 \\
\hline & Total & 224 & 279 & 234 & 201 & 385 & 297 \\
\hline \multirow{4}{*}{ Flattop 23} & ${ }^{233} \mathrm{U}$ & 167 & 180 & 156 & 218 & 304 & 227 \\
\hline & ${ }^{235} \mathrm{U}$ & 5 & 7 & 5 & 16 & 17 & 13 \\
\hline & ${ }^{238} \mathrm{U}$ & 41 & 58 & 46 & 53 & 45 & 36 \\
\hline & Total & 171 & 189 & 163 & 225 & 308 & 231 \\
\hline \multirow{3}{*}{ Big-ten } & ${ }^{235} \mathrm{U}$ & 456 & 575 & 441 & 43 & 200 & 132 \\
\hline & ${ }^{238} \mathrm{U}$ & 189 & 273 & 217 & 218 & 400 & 307 \\
\hline & Total & 493 & 637 & 491 & 218 & 448 & 334 \\
\hline \multirow{4}{*}{ ZPPR-9 } & ${ }^{235} \mathrm{U}$ & 6 & 7 & 5 & 14 & 13 & 9 \\
\hline & ${ }^{238} \mathrm{U}$ & 76 & 103 & 81 & 45 & 16 & 11 \\
\hline & ${ }^{239} \mathrm{Pu}$ & 331 & 371 & 332 & 706 & 639 & 520 \\
\hline & Total & 340 & 385 & 342 & 708 & 639 & 521 \\
\hline
\end{tabular}

detailed covariance matrices available in some nuclear data evaluation (JENDL-4.0, SCALE-6, etc.) was possible. Table 4 compares the uncertainties in $k_{\text {eff }}$ and $\beta_{\text {eff }}$ calculated using the above "two-block" PFNS covariances with those based on the PFNS covariances from JENDL4.0 and SCALE-6.0. In spite of its simplicity the procedure is shown to predict similar uncertainties, both for $k_{\text {eff }}$ and $\beta_{\text {eff }}$ uncertainties, as the more sophisticated methods used in the JENDL-4.0 and SCALE-6.0 covariance data evaluations. This good agreement can be explained by the relatively narrow-energy sensitivity of the $k_{\text {eff }}$ and $\beta_{\text {eff }}$ to the fission spectra.

A similar procedure could be temporary applied to evaluate the SED uncertainties for other reactions such as (in)elastic scattering, until more sophisticated evaluations become available. 


\section{Conclusions}

The availability of the covariance data improved over the last decades. Experience in using covariance matrices of different origin (such as JEFF, JENDL, ENDF, TENDL, SCALE, etc.) any types (MF31, MF33, MF34 and MF35) in sensitivity and uncertainty analysis of vast list of cases ranging from fission to fusion and from criticality, kinetics and shielding to adjustment applications is presented. The status of the available covariance and future needs in the areas including secondary angular and energy distributions is addressed. Of particular concern is the lack of correlation among different isotopes and reactions, the differences among the recent covariance matrices although the cross-section evaluations are mostly based on similar experimental data, and the lack of covariance information for some more specific reactions and reaction types (such as e.g. SAD/SED).

The authors acknowledge the financial support from the Slovenian Research Agency (research core funding No. PR-07382). Part of the work has been carried out within the framework of the EUROfusion Consortium and has received funding from the Euratom research and training programme 2014-2018 under grant agreement No. 633053. The views and opinions expressed herein do not necessarily reflect those of the European Commission.

\section{References}

1. S.C. van der Marck, Benchmarking ENDF/B-VII.1, JENDL4.0 and JEFF-3.1.1 with MCNP6, Nucl. Data Sheets 113, 2935 (2012)

2. K. Shibata et al., JENDL-4.0: a new library for nuclear science and engineering, J. Nucl. Sci. Technol. 48, 1 (2011)

3. I. Kodeli, Multidimensional deterministic nuclear data sensitivity and uncertainty code system: method and application, Nucl. Sci. Eng. 138, 45 (2001)

4. I. Kodeli, S. Slavič, SUSD3D computer code as part of the XSUN-2017 windows interface environment for deterministic radiation transport and cross section sensitivity uncertainty analysis, Sci. Technol. Nucl. Install. 2017, 16 (2017)

5. I. Kodeli, Cross section sensitivity analysis of $14 \mathrm{mev}$ neutron benchmark experiment on Tungsten, J. Nucl. Mater. 329-333, 717 (2004)

6. U. Fischer, P. Batistoni, A. Klix, I. Kodeli, R. L. Perel, Neutronics R\&D efforts in support of the European breeder blanket development programme, Nucl. Fusion 49, 065009 (2009)

7. P. Batistoni, M. Angelone, U. Fischer, A. Klix, I. Kodeli, D. Leichtle, M. Pillon, W. Pohorecki, R. Villari, Neutronics experiments for uncertainty assessment of tritium breeding in HCPB and HCLL blanket mock-ups irradiated with $14 \mathrm{MeV}$ neutrons, Nucl. Fusion 52, 083014 (2012)

8. M. Angelone, U. Fischer, D. Flammini et al., Neutronics experiments, radiation detectors and nuclear techniques development in the EU in support of the TBM design for ITER, Fusion Eng. Des. 96-97, 2 (2015)

9. I. Kodeli, K. Kondo, R.L. Perel, U. Fischer, Cross-section sensitivity and uncertainty analysis of the FNG copper benchmark, Fusion Eng. Des. 109-111, 1222 (2016)
10. I. Kodeli, L. Snoj, Evaluation and uncertainty analysis of the KRITZ-2 critical benchmark experiments, Nucl. Sci. Eng. 171, 231 (2012)

11. I. Kodeli, Sensitivity and uncertainty in the effective delayed neutron fraction $\left(\beta_{\text {eff }}\right)$, Nucl. Instrum. Methods Phys. Res. A 715, 70 (2013)

12. P. Romojaroa, F. Alvarez-Velarde, I. Kodeli et al., Nuclear data sensitivity and uncertainty analysis of effective neutron multiplication factor in various MYRRHA core configurations, Ann. Nucl. Energy 101, 330 (2017)

13. I. Kodeli, D.L. Aldama, P.F.A. de Leege, D. Legrady, J.E. Hoogenboom, P. Cowan, Multigroup coupled neutron-gamma cross-section library for deterministic and Monte Carlo borehole logging analysis, Nucl. Sci. Eng. 157, 210 (2007)

14. R.E. MacFarlane, D.W. Muir, The NJOY Nuclear Data Processing System Version 99 (RSICC Code Package PSR368, LA-12740-M, Los Alamos National Laboratory, 1999)

15. I. Kodeli, ANGELO-LAMBDA, Covariance Matrix Interpolation and Mathematical Verification, NEA-DB Computer Code Collection, NEA-1798/02, 2008

16. ZZ-SCALE6/COVA-44G, PACKAGE-ID: USCD-1236/02 (May 2009), USCD-1236/03, May 2012

17. C.J. Diez et al., Comparison of nuclear data uncertainty propagation methodologies for PWR burn-up simulations, Ann. Nucl. Energy 77, 101 (2015)

18. D. Rochman, O. Leray, A. Vasiliev, H. Ferroukhi, A.J. Koning, M. Fleming, J.C. Sublet, Bayesian Monte Carlo method for fission yield covariance information, Ann. Nucl. Energy 95, 125 (2016)

19. S. Tarride, I. Kodeli, K.-H. Schmidt, P. Dossantos-Uzarralde, in Proceeding 26th International Conference of Nuclear Energy for New Europe (NENE-2017), Bled, Sept. 2017

20. K.-H. Schmidt, B. Jurado, C. Amouroux, General Description of Fission Observables (Pergamon, 2014)

21. A. Aures, F. Bostelmann, I.A. Kodeli, K. Velkov, W. Zwermann, Uncertainty in the delayed neutron fraction in fuel assembly depletion calculations, EPJ Web Conf. 146, 02052 (2017)

22. K. Furuta, Y. Oka, S. Kondo, A cross-section sensitivity and uncertainty analysis on fusion reactor blankets with SAD/ SED eff ects, Nucl. Eng. Des. Fusion 3, 287 (1986)

23. V. Pronayaev, S. Tagesen, H. Vonach, S. Badikov, Improvement of the EFF-2 evaluations for ${ }^{52} \mathrm{Cr} ;{ }^{56} \mathrm{Fe},{ }^{58} \mathrm{Ni}$ and ${ }^{60} \mathrm{Ni}, \mathrm{EFF}-\mathrm{DOC}-377,1995$

24. H. Vonach, S. Tagesen, M. Wagner, A. Pavlik, Final Report for Contact nr. 395-89-8/FU/D/NET, EFF-DOC-85, 1991

25. I. Kodeli, Computational Tools for Cross-Section Sensitivity and Uncertainty Evaluation. Final Report on Task EFFBB4-F, EFF-DOC-446, 1996

26. M.B. Chadwick, M.W. Herman, P. Obložinský, et al., ENDF/ B-VII.1 nuclear data for science and technology: cross sections, covariances, Nucl. Data Sheets 112, 2887 (2011)

27. A.J. Koning, D. Rochman, J. Kopecky et al., TENDL- 2015 available from https://tendl.web.psi.ch/tendl_2015/ tendl2015.html

28. I. Kodeli, A. Trkov, R. Capote, Y. Nagaya, V. Maslov, Evaluation and use of the prompt fission neutron spectrum and spectra covariance matrices in criticality and shielding, Nucl. Instrum. Meth. Phys. Res. A 610, 540 (2009) 